\title{
Becoming Human? Ableism and Control in Detroit: Become Human and the Implications for Human- Machine Communication
}

\author{
Marco Dehnert ${ }^{1}$ (D) and Rebecca B. Leach ${ }^{1}$ (D) \\ 1 The Hugh Downs School of Human Communication, Arizona State University, Tempe, AZ, USA
}

\begin{abstract}
In human-machine communication (HMC), machines are communicative subjects in the creation of meaning. The Computers are Social Actors and constructivist approaches to HMC postulate that humans communicate with machines as if they were people. From this perspective, communication is understood as heavily scripted where humans mindlessly apply human-to-human scripts in HMC. We argue that a critical approach to communication scripts reveals how humans may rely on ableism as a means of sense-making in their relationships with machines. Using the choose-your-own-adventure game Detroit: Become Human as a case study, we demonstrate (a) how ableist communication scripts render machines as both less-than-human and superhuman and (b) how such scripts manifest in control and cyborg anxiety. We conclude with theoretical and design implications for rescripting ableist communication scripts.
\end{abstract}

Keywords: human-machine communication, ableism, control, cyborg anxiety, Computers are Social Actors (CASA)

\section{Introduction}

Human-Machine Communication (HMC) refers to both a new area of research and concept within communication, defined as "the creation of meaning among humans and machines" (Guzman, 2018, p. 1; Fortunati \& Edwards, 2020). HMC invites a shift in perspective where

CONTACT Marco Dehnert (D) - The Hugh Downs School of Human Communication - Arizona State University • P.O. Box

871205 • Tempe, AZ 85287-1205, USA • marco.dehnert@asu.edu 
technology is no longer a tool or the medium for communication, but a communicator itself (Guzman, 2018). This has far-reaching implications for the communication discipline inasmuch as "HMC 'frees' the machine from its relegation to the role of medium and, as a result, communication itself is loosed from a definition based on the ontology of participants" (Guzman, 2018, pp. 17-18). As such, "the machine has become a communicative subject" (Guzman, 2018, p. 12). Yet, the question remains, how do we treat that communicative subject? The Computers are Social Actors (CASA) approach postulates that humans fundamentally understand and relate to computers, machines, and technologies as if they were other people (Nass \& Moon, 2000). CASA has been one of the most influential perspectives in HMC because it serves as a theoretical foundation for understanding how machines are treated as a communicative subject in human-machine interactions. The CASA approach applies an understanding of communication as heavily scripted, such that communication is a largely automatic process where certain triggers activate communication scripts in a "click, whirr" fashion (Cialdini, 2009; Westerman et al., 2020). Complementing this scripted approach, A. Edwards et al. (2019) propose a constructivist research paradigm in HMC wherein "people seek to employ the same judgement and interaction patterns developed for use with other people in their communication with machine partners" (p. 312). These lines of scholarship have demonstrated that various communication theories originally formulated for human-human interactions can also provide valuable insights in the humanmachine context (Fortunati et al., 2020; Westerman et al., 2020). We contend, however, that critical communication perspectives have been largely absent from this work. Building upon previous work that explores the scripted nature of communication in HMC, we argue that a critical perspective is a promising avenue for a deeper interrogation of how humans make sense of their interactions with machines.

A critical perspective on a constructivist research paradigm in $\mathrm{HMC}$ attunes us to the ubiquitous yet powerful societal systems of oppression that guide communication scripts: structures such as heteronormativity and Whiteness that have been intensively theorized by critical communication scholars. Among these pervasive social scripts, we hold that ableism may be an alluring but dangerous script humans may rely on to make sense of differences between humans and machines, in spite of (or arguably because) machines are becoming increasingly human-like. As a system of beliefs, practices, and processes, ableism refers to the cultural construction of a "normal" body and conceives of any deviation from that "normal" body as lack thereof-be it in the form of disability or extraordinary abilities (Cherney, 2019). In this essay, we are not so much concerned about "actual" ontological differences between humans and machines (e.g., A. P. Edwards, 2018; Guzman, 2020), but rather how a constructivist approach to HMC illustrates the ways humans perceive and construct machines as communicative subjects. Although different abilities between humans and machines are oftentimes a matter of design and technology, an ableist framework highlights how different cap/abilities are constructed and interpreted culturally. As humans' interactions with social robots are likely going to increase in the near future, it is imperative to evaluate the risk attached to ableist understandings of the differences in machines' technological cap/abilities. In particular, an ableist framework may result in foreclosing authentic human engagement with machines or lead to the exclusion and rejection of machines, 
akin to society's treatment of disabled people (Johnson \& Kennedy, 2020). ${ }^{1}$ We argue that as machines are simultaneously rendered as both less-than-human/disabled (lacking technological cap/abilities and/or "true" emotions) and more-than-human (increased processing power and physical cap/abilities of machines), humans may be tempted to quickly characterize machines through the cognitive shortcut of ableism. In accordance with a constructivist approach in HMC, we explore the implications of how ableist communication scripts may influence how humans make sense of machines and thereby make sense of themselves.

This paper begins with a review of CASA and constructivist literature in HMC, noting the ways in which a critical perspective can supplement current understandings. We especially highlight how a posthuman and critical approach to disability "allows us to think across binaries of self/other, nature/technology and human/machine" (Goodley et al., 2014, p. 348). To illustrate our claims and highlight how ableist communication scripts may play out in human-machine interactions, we turn to the case of Detroit: Become Human (DBH; Quantic Dream, 2018), an android-centered video game that invites the player to develop parasocial interactions with android characters (Craig et al., 2020, Leach \& Dehnert, in press) and explore what it means to be(come) human in a world full of humanoid machines. In $D B H$, androids face ableist discrimination both for being less-than-human and superhuman at the same time, exerted through various types of control and fueled by cyborg anxiety (i.e., the fear that the performance and embodiment of a disabled person exposes the porous and permeable boundaries of what it means to be an able-bodied and ableminded human). Finally, we conclude with future directions for research in both HMC and human-human communication (HHC) contexts.

\section{Mobilizing a Critical Perspective in Human-Machine Communication}

With a focus on the meaning-making processes between humans and machines, one of the central tenets in HMC is arguably the ontological shift for communication technology (e.g., robots, algorithms, artificial intelligence [AI], etc.) from channel to communicator (Gunkel, 2012; Guzman, 2018; Peter \& Kühne, 2018). In other words, rather than operating as the medium in HHC that people talk through, the machine takes on the role of communicator that people talk with. Understanding the machine as a communicative subject raises the question of how humans engage with this communicative other. Ontological differences between humans and machines play an important role in this regard (A. P. Edwards, 2018; Gunkel, 2012; Guzman, 2020; Sandry, 2018), but we can also examine how humans treat and relate to machines as if they were other people through a Computers are Social

\footnotetext{
1. As communication scholars, we are sensitive of the words and terms we use and thus deliberately use phrases such as "the disabled," "disabled people," and "nondisabled people." Rather than the common person-first approach, we see this choice as a "rhetorical practice that challenges ableist rhetoric" (Cherney, 2019, p. 24). Critical and posthuman disability studies take a social model framework to disability, where disability is understood as a culture (or multiple cultures). As Makkawy and Moreman (2019) poignantly note, "Like the cultural signifiers of Latinx and even queer, we would not say person of Latinx or person of queerness" (p. 414n1, emphasis in original). Finally, our use of the terms "disabled people" and "nondisabled people" foregrounds "the way that society strips humanity from the disabled through oppressive ableist practices" (Cherney, 2019, p. 24).
} 
Actors (CASA) framework (Nass \& Moon, 2000). Importantly, CASA does not apply to every machine or technology. Instead, CASA focuses on media agents: "any technological artifact that demonstrates sufficient social cues to indicate the potential to be a source of social interaction" (Gambino et al., 2020, p. 73). Essentially, CASA posits that people are likely to apply social scripts from HHC when interacting with machines that possess social cues and function as communicators (Krämer et al., 2012; Nass \& Moon, 2000).

From a CASA perspective, communication is viewed as heavily scripted. Based on previous interactions and experiences, scripts function as mental representations or heuristic shortcuts to make sense of situations, interactions, or people, and these scripts are perpetually reified through ongoing interactions (Cialdini, 2009; Nass \& Moon, 2000). CASA holds that humans utilize communication scripts developed in an HHC context when interacting with machines. This is conceptualized as a mindless process as people use social cues of machines as a heuristic to resort to learned and constantly reified social scripts in the moment of interaction (Nass \& Moon, 2000). Given that people often apply the same social scripts used with human communicators to machines, theories that explain these HHC processes should be applicable to HMC, such as interpersonal and computermediated communication theories (see Westerman et al., 2020, for an overview). HMC has also seen a turn toward a constructivist approach to human-machine interactions. A constructivist perspective holds that "people accumulate and integrate communication knowledge, tendencies, and abilities through previous interactions, and that those form the basis of later social cognitive and message construction behaviors" (A. Edwards et al., 2019, p. 312). From a constructivist perspective, communication scripts applied to HMC are the result of previous interactions and exposure to discourse about machines, where "our social interactions unfold first and foremost through a process of prototyping the potentially communicative other" (p. 312). Together, constructivism and CASA explain the ways in which humans treat machines as if they were people by applying communication scripts that are continually (re)constructed through discourse about and interactions with machines.

While the move toward a constructivist paradigm has transformed understandings of both HMC and HHC (Westerman et al., 2020), we contend that critical communication perspectives have been largely absent from these conversations. Critical perspectives in communication seek not only to understand the undergirding structures that guide human communication (i.e., scripts), but also how these scripts are indicative of power and social hierarchies. Additionally, critical approaches are united by an emancipatory impetus striving for social change. From a critical perspective, social scripts are borne from larger systems of belief (e.g., racism, sexism, or ableism) that guide people's worldview and interactions. Moreover, while humans and machines should be considered as different (humans are not machines) but complimentary (both are inherently valuable for different reasons), a critical perspective shows that differences are often cast in binaries where one form of identity is rendered as normal and desirable (e.g., Whiteness, masculinity, able-bodiedness) and other expressions of identity are cast as abnormal and deviant (e.g., Black, Indigenous, and people of color, femininity, disability). These scripts focused on difference apply even to human-machine interactions. In this paper, we focus specifically on ableism and how this lens generates scripts that guide HMC and sensemaking. As machines become more human-like, ableism may be a tempting script humans mindlessly rely on to make sense of the technological and design differences between humans and machines. 


\section{Ableism: The Posthuman Machine and Cyborg Anxiety}

We argue that bringing ableism in conversation with HMC is worthwhile, given that "the core of ableism is an idealized norm that defines what it means to be human" (Cherney, 2019 , p. 8). Broadly speaking, ableism is a societal structure, a network of beliefs, communication practices, and institutional processes that describes a corporeal standard. Ableism casts an idea of a "normal" body and mind and ultimately speaks to the question of what it means to be human (cf. Van Trigt et al., 2016). Traditional ideologies surrounding ableism support an "ableist conflation" that equates disabilities to pain and suffering (Reynolds, 2017). Extending this logic, an ableist discourse posits that the only life worth living is one lived by the nondisabled, and that those who are disabled deserve either pity or contempt. Disability studies scholars consistently show how the economic, political, and social condition of neoliberalism demands an abled, normative corporeal standard (e.g., Goodley, 2014; McRuer, 2006). From a neoliberal perspective, bodies must be flexible and productive (read: neither disabled nor extraordinarily abled) in order to meet the strenuous requirements of neoliberal capitalism. When a society privileges such a discourse, the nondisabled are more likely to engage in communicative processes that otherize the disabled, which can be an intentional or unintentional choice (Mik-Meyer, 2016). One way in which ableist discourses play out is through different types of control, as we explore in more detail below.

More recently, critical disability studies aim to deconstruct the disabled/abled binary and integrate perspectives from postcolonial, queer, and feminist theories into the study of disability (Goodley, 2014; Goodley et al., 2019). Goodley et al. (2014) explicitly align the project of critical disability studies with posthuman thought and argue that "disability is the quintessential posthuman condition" (p. 348, emphasis in original). Albeit valuable critiques of posthuman and critical disability studies that center mostly on its ethical and political applicability (e.g., Meekosha \& Shuttleworth, 2009; Vehmas \& Watson, 2014), we utilize this perspective as it works the disabled/abled binary, shifts the focus from the disabled body to the ideological system of ableism, and thus has "an antithetical attitude towards the taken-for-granted, ideological and normative under-girdings of what it means to be a valued citizen of society" (Goodley et al., 2014, p. 348). As a critique of humanism's anthropocentrism, Van Trigt et al. (2016) argue for "understand[ing] the posthuman condition as one in which we constantly reflect on humanity rather than as a condition beyond humanity" (p. 126). Thus, quite similarly to HMC's critique of the anthropocentricity of communication, "disability allows us to think across binaries of self/other, nature/ technology and human/machine" (Goodley et al., 2014, p. 348), thereby decentering the human.

Our goal is not to frame ableism as an analytic for explaining actual ontological differences between humans and machines, which are often justified by technological limits or design choices. Rather, we propose ableism as one societal structure that undergirds humans' communicative scripts that may be applied from a human-human context to make sense of HMC and to judge the communicative machine-other. In short, we seek to complicate constructivist and CASA approaches to HMC from a critical perspective. For instance, some children have been shown to see the robot DORO as a child younger than themselves or as disabled (Fortunati et al., 2020). As such, our approach is distinct from what has been articulated as "technoableism" (Shew, 2020) or "crip technoscience" (Hamraie \& Fritsch, 
2019). These perspectives theorize the technological enhancement of disabled people and must grapple with ableist discourses of "overcoming" or "curing" disability, oftentimes from a transhumanist perspective (Wolbring, 2009). Common examples in this view of ableism and technology are cochlear implants, exoskeletons, or proposed cures for autism (Shew, 2020; Wolbring, 2009). One key concept of relevance to our project that is theorized at the intersections of dis/ability and technology is Donna Haraway's cyborg.

While the usefulness and appropriateness of Haraway's cyborg theory for disability studies has been widely contested in the field (e.g., Reeve, 2012), the notion of a cyborg anxiety proves useful as it relates to the perception of a disabled person as cyborg. Again, we are not so much concerned with ontology (i.e., whether a person/machine is a cyborg), but rather with perceptions and constructions of ontological difference (i.e., whether a person/machine is perceived as a cyborg). Cyborg anxiety describes a fear that the body and performance of a disabled person "expose the weakness in tenuous and arbitrary definitions of what it means to be human" (Cherney, 2019, p. 121), and calls into question "something quite fundamental, quite invisible, about the boundaries between disabled and non-disabled groups" (Swartz \& Watermeyer, 2008, p. 188). Notably, cyborg anxiety accounts for disability not only as lacking ability from the "normal" body but also for those bodies who exceed this corporeal standard in some "extraordinary" or even "superhuman" way. As Cherney (2019) poignantly writes, "In the ableist mindset, having only one arm or three pose the same problem" (p. 9). Therefore, while technological shortcomings in machines may be interpreted as less-than-human via ableist scripts, machines' technological advances (e.g., superior cognitive processing power, enhanced knowledge due to strong algorithmic search engines, or increased strength and stamina as compared to humans) may render them superhuman. These varying levels of abilities between machines and humans do not automatically or ontologically indicate machines' position as either less-than-human or superhuman. Rather, as we further highlight in our following analysis of $D B H$, we contend that the application of ableist human-to-human scripts to human-machine contexts may lead to such a reading as humans make sense of their ever-changing relationships with technology.

\section{An Introduction to Detroit: Become Human}

Although various media have explored the narrative drama and tension between human and machine (e.g., Humans, West World, Her, Nier Automata), we have selected DBH as our case study due to its highly interactive nature as a choose-your-own-adventure video game. The level of interactivity within video games acts as a strong catalyst in the development of parasocial relationships with media characters and encourages players to nurture a sense of identification with the media characters' personalities and plights (Christoph et al., 2009). $D B H$ is uniquely positioned to not only illustrate how ableist scripts form as a means of sense-making for human-machine relationships; additionally, $D B H$ provides players the opportunity to actively engage in the creation and resistance of such scripts as the player in a hypothetical yet strangely familiar world. $D B H$ requires players to recognize their real-life biases and values regarding what it means to be human versus a machine as they make in-game decisions that affect the outcome of the narrative (Leach \& Dehnert, in press). Finally, video games such as $D B H$ are important contributors to larger discourses such as ableism and will thus likely influence expectations of 
and communication scripts for actual human-machine encounters (Craig et al., 2020; A. Edwards et al., 2019).

$D B H$ takes place in a futuristic version of Detroit in 2038 where humanoid androids are common household goods. Different models of androids possess varied functions, but most androids are designed to assist with household chores (e.g., cleaning, cooking, babysitting, etc.). Even the most basic androids excel in performance and adaptive learning, clearly designed to please their human masters. In this society, androids are conventionally attractive, eternally youthful, and intelligent. Despite possessing these coveted features, androids are constantly treated as less-than-human and undeserving of basic human rights. At best, androids are treated as valuable products to maintain a pleasant home. At worst and far more common in the narrative of $\mathrm{DBH}$, androids are frequent victims of physical, verbal, and emotional abuse. Androids are also blamed for societal problems, including rising unemployment rates.

The crux of all this mistreatment against androids lies in the argument that androids lack souls and the ability to feel emotions. However, as players of $D B H$ follow the narratives of three androids, it quickly becomes clear that androids have the capacity to genuinely experience emotion both for humans and for fellow androids. In this choose-your-ownadventure game, the player cycles between the stories of three android characters: Connor, Markus, and Kara. Connor is a prototype detective android who assists the Detroit police in investigating cases of android deviancy-androids who are considered "deviant" because they have broken their programming by seemingly gaining sentience and emotion. Another one-of-a-kind prototype, Markus was designed to be a caretaker for an elderly artist. Unlike most androids featured in $\mathrm{DBH}$, Markus comes from a loving and respectful home, but unexpected tragedy forces Markus out of his home and eventually places him on the path to become the leader of a massive android resistance against human control. Finally, Kara is a female android employed to be a basic caretaker for a young child named Alice, but her story also quickly escalates as Kara decides to save Alice from an abusive home. Depending on the player's choices in this game, the stories of these three main characters may intersect and meaningfully impact one another, which can result in positive outcomes like unexpected alliances or negative outcomes such as permanent character deaths.

\section{An Ableist Discourse to Control Androids}

While androids in $\mathrm{DBH}$ are perceived to be superhuman in most regards (strength, beauty, intelligence, and function), they are perceived as less-than-human due to their lack of emotions. As Connor repeatedly mentions, "I'm a machine designed to accomplish a task." Androids were not designed to have "true" emotions or a soul; they were designed to satisfy their owner's needs in any way, from performing household chores to even acting as sexual partners. The built-in power hierarchy between humans as "masters" and machines as "slaves" resembles the perspective of early cybernetics and engineering fields that view technology as tools, where "human-machine communication within this context is a process of interacting with technology, a tool, as to leverage it for some purpose" (Guzman, 2018, p. 7). The ableist discourses that humans use to make sense of machines' different cap/abilities render androids in $\mathrm{DBH}$ as inherently lacking and anxiety-inducing-thereby modeling, as we argued earlier, such discourses outside of $\mathrm{DBH}$. 
In $D B H$, Detroit in 2038 is a society that draws heavily on ableist scripts to create clear material and discursive boundaries between superior humans and inferior androids. Correspondingly, Guzman (2020) found that humans conceptualize the ontological boundaries between humans and machines along several interrelated divides, including (a) origin of being (humans come from nature, while machines are created by humans), (b) tools (machines) as well as tool users and creators (humans), (c) autonomy (humans have free will, while machines are limited by their programming), (d) intelligence (humans excel at some tasks, while machines excel at others), and (e) emotion (humans feel emotions, while machines do not). The ableist discourses in $\mathrm{DBH}$ play out along those ontological divides and cast the androids as tools, rather than communicators. This shift from androids as tools to androids as communicators requires a peaceful or violent android revolution, depending on the player's choices, in which androids claim rights similar to human rights to be treated equally.

Nonetheless, even if we reformulate our understanding of machines and move away from seeing them as the medium to seeing them as a communicative subject, ableism is still at play. If ableism defines any deviation from a constructed norm as deficiency (Cherney, 2019), it casts an idea of a "normal" body and ultimately speaks to the question of what it means to be human. Cherney identifies and explores three rhetorical warrants of ableist culture that, together, form its normative force: deviance is evil, normal is natural, and body is able. Androids in $\mathrm{DBH}$ violate all three warrants within the course of the game, rendering them as evil and lacking from an ableist perspective. By this logic, androids that gain sentience should qualify as "human," but this fictional society fueled by cyborg anxiety (Swartz \& Watermeyer, 2008) instead finds new ways of rewriting ability such that androids' superhuman qualities also violate the norm. To maintain power distance between themselves and the technological other, humans reconstruct ableist scripts to treat the extraordinary as a deviation (similar to disability). This allows humans to continue to marginalize and oppress androids, as evidenced in ableist microaggressions, dehumanizing use of pronouns, and violent action against androids. This forecloses any possibility for treating machines as communicative others.

Within minutes of starting a game of $D B H$, players learn that they are playing within a fictional world featuring strong anti-android ideals and rhetoric. The opening scenes of the game confirm this grim reality by highlighting a variety of injustices against androids, including: Markus being harassed by human protestors, Connor being vehemently dismissed as a "a piece of plastic" by everyone around him, and Kara being berated and beaten by her owner. In fact, androids are treated like other historically oppressed minorities in the U.S. (e.g., Markus, the only Black playable character in $D B H$, has to stand in the back of the bus away from humans). These scenes are undoubtedly meant to stir feelings of shock and discomfort in players. Unlike the unseen players, however, the three main characters simply ignore or submit to these various injustices at the beginning of the game. The injustices in androids' lives may be evident to players, but the characters of $\mathrm{DBH}$ are initially resigned and even blind to their unfair treatment. It is not until later that the androids have the opportunity to awaken to their circumstances and resist against the forces that control them.

$D B H$ may take place in a fictional setting, but the struggles its characters face reflect the same hegemonic processes that exist in reality. On the surface, the dominant discourse 
in $\mathrm{DBH}$ is anti-android and thus may seem irrelevant to our society where we have yet to advance to a stage where androids are common household features. However, the antiandroid discourse in $\mathrm{DBH}$ only masks the true discourse at play: an ableist discourse that purposefully privileges the nondisabled and suffocates the minority group of the disabled. By analyzing how human characters in this fictional Detroit mindlessly apply ableist humanto-human scripts when interacting with machine-others to make sense of difference, we may be better equipped to recognize how such ableist human-to-human scripts echo in our own society. Further, as part of larger discourses surrounding androids, video games like $D B H$ shape discourses about machines outside of the game as well. Importantly, then, $D B H$ provides opportunities to dissect the overt and covert ways that dominant groups control minority groups (e.g., androids as disabled) to support their preferred status quo. The following section examines the communicative scripts that comprise the ableist discourse in $\mathrm{DBH}$ and the various forms of control that support and maintain it.

\section{Maintaining the Status Quo: Types of Control}

Humans are the social actors that produce and reproduce the social structures and discourses that guide society today (Giddens, 1984). Through careful observation and reflection, it is possible for players to determine how the dominant group of this fictional society (i.e., humans) strategically defines "ability" to its own benefit and to the detriment of the marginalized group (i.e., androids). Whereas technological cap/abilities may be the main difference between humans and machines in reality, the society in $\mathrm{DBH}$ positions emotion as the most important ability of all-the critical ingredient of being human. However, when androids display signs of emotional intelligence and sentience, the ableist discourse that humans have constructed is suddenly no longer as beneficial as it seems. If androids possessed the ability to feel emotion, the ableist discourse that is fundamentally tied to emotion would no longer be able to separate humans and androids into better-than and less-than camps, respectively.

To maintain a status quo at the level presented in $D B H$ (i.e., a society where humans are powerful and androids are unquestionably considered "less-than"), proponents of the ableist discourse must find ways to maintain control over the minority group. $D B H$ 's rich narratives showcase control in a wide variety of forms, reflecting the same types of control that are used outside of the game to oppress weaker, marginalized groups. Broadly, these types of control can be categorized into obtrusive and unobtrusive control. Obtrusive control consists of any overt attempt to direct others' actions, whereas unobtrusive control is the process where these attempts are subtle and essentially unseen by the manipulated members (Tompkins \& Cheney, 1985). Importantly, unobtrusive control is only possible when a group strongly identifies with the larger organization's values and actively contributes to the organization's success, even when it does not benefit themselves (Tompkins \& Cheney, 1985). In the context of $D B H$, androids are controlled in both obtrusive and unobtrusive ways to support the pre-existing ableist discourse.

Obtrusive forms of control are often the easiest to identify because they are explicit in function and intention. Three of the most well-known types of control are from R. C. Edwards's (1978) conceptualization of control: simple control (a superior or manager provides a direct order to a subordinate), technical/technological control (machines or 
technology substitute for a supervisor and guide action), and bureaucratic control (rule systems that guide subordinates' performance and decision-making). Although some scholars argue about which of these three types of control can function obtrusively or unobtrusively (Gossett, 2009), we contend that $D B H$ characterizes these three controls as explicit and obtrusive. We further argue that control is enacted through recourse to ableist communication scripts in humans interactions with machines. Simple control is a mainstay in daily interactions between humans and androids because androids are programmed to follow their owners' orders without question. Technical control is thus the underlying mechanism that allows for simple control to be powerful. Androids are extremely aware of the technical restrictions imposed by their programming, such that they cannot act in ways that conflict with humans' orders. Interestingly, there are also multiple cases where simple and technical control are in tension with one another, resulting in one overriding the other. Connor is often placed in this position throughout the game, where the purpose of his technical control (i.e., his programming to be an efficient detective) conflicts with Hank's simple control of Connor. As his human partner, Hank has the authority to tell Connor what to do (e.g., "stay in the car," "don't approach the crime scene"), but the player as Connor is allowed to choose between following the expectations of technical control or simple control in a small moment of veiled freedom. Here, the player experiences firsthand the contradicting tensions exerted by simple and technical control on the android body. The game leaves it open for the player to literally play with different responses to this dilemma of control.

Technical control is arguably the greatest form of obtrusive control for androids. In fact, in order to become "deviant," androids must break the code of their programming to gain free will. All three main characters in the game have the opportunity to become deviant, which requires tearing down or breaking a wall of code that only androids can see. Kara is the first main character who receives the opportunity to break her code, at which point she is no longer subject to technical control and simple control. Similarly, Markus breaks free from his code to protect himself and his owner. Without technical control in action, simple control loses its power as well. However, resisting bureaucratic control is notably difficult, given that rule systems are represented in the structures that humans create and maintain through communicative scripts. Even when Kara gains free will, she still struggles to function in a world of bureaucratic control. For example, when Kara escapes with Alice and looks for shelter at a hotel, she is rejected on the grounds that she is an android. Similarly, Connor's existence as a detective android is heavily embedded in bureaucratic control because the police's rule system states that he is unable to perform his job without a human supervisor nearby. This is why Connor often searches for Hank in various locations before beginning an investigation-not because he necessarily wants a partner, but because he is required to have Hank nearby to perform his work.

The restrictions associated with obtrusive control are certainly problematic and constitute a major obstacle for the androids that desire a revolution in the game. Indeed, much of Markus's narrative focuses on overcoming obtrusive control, as evidenced by his efforts to release other androids from technical control, breaking into stores to save his fellow androids, and leading protests even in the face of human police suppression. With its choose-your-own-adventure design, the game leaves it up to the player to decide exactly how to resist these various types of control. For instance, the player as Markus can either lead a peaceful protest or choose to detonate a nuclear bomb in the face of ongoing violent 
responses from humans. Despite the importance of such efforts, the greatest obstacles are arguably the ones related to unobtrusive control. After all, in the history of this fictional world, androids have endured oppressive scripts and obtrusive control for so long because they believed in their organization's values (e.g., human life is precious) and they believed their role in society to serve humans was a critical part of their existence and mission. This faith in society's values was so strong that androids unwittingly embraced the ableist discourse that humans constructed.

Evidence of unobtrusive control exists in all aspects of android life in $D B H$, both in discursive and material forms. In language, humans refer to androids with "it" pronouns to clearly dehumanize them. Additionally, androids have moved past the uncanny valley (cf. Mori, 2012) and are so convincingly human that they have to self-identify as androids by wearing a uniform that reads "ANDROID" on their back and their model number on their chest. Additionally, androids have a glowing mark on their temple. These seemingly small markers reinforce the idea that androids are "others" and "less-than." Androids also enact concertive control over each other to protect the same values that oppress them, sometimes acting as faux superiors over one another when a fellow android is displaying deviancy (Wright \& Barker, 2000). Connor's existence as a detective android speaks to such concertive control, because he hunts fellow androids to uphold human values. Players also sometimes see the enduring results of unobtrusive control in deviant androids. Perhaps most notably, a grim moment occurs when Kara eventually discovers that Alice (the child she saved and spent the whole game protecting) is actually an android and not a human like she thought. Kara's shock and sadness are fleeting but noticeable, prompting her companion, Luther, to ask her if it mattered if Alice was human or not. Based on the player's choices, Kara has the potential to overcome this realization and move forward to a life with Alice as her child. This interaction gives players a brief glimpse of the enduring effects of unobtrusive control through ableist scripts on Kara, who had her free will but still believed in the values she always possessed-that relationships with humans were precious and more important than the relationships she could build with her fellow androids.

This case study illustrates the ways in which communicative scripts are deeply embedded in cultural discourses like ableism and how they can manifest in issues of control. By inviting player engagement with ableist discourses, $D B H$ reveals how humans may approach HMC through ableist communication scripts that guide their sensemaking of machines. As part of larger societal discourses, video games such as $D B H$ arguably shape the ways in which we think about machines. Moreover, by giving players an opportunity to affect the game's narrative, $D B H$ opens up space for resisting and reworking ableist scripts. Although communicative scripts may exude a sense of stability, they are also in need of constant reification to maintain their force. Interrogating the instability of ableist scripts invites the possibility for rescripting, or the rewriting of communicative scripts through changed action (Moreman \& McIntosh, 2010).

\section{Concluding Thoughts and Future Directions}

In this paper, we have argued that the application of HHC scripts to human-machine interactions requires a critical perspective on the scripts being used to make sense of the interactions and identities of both humans and machines. By describing how ableism is 
a particularly appropriate framework in the context of HMC-because humans may fall back on ableist frameworks to make sense of cap/ability differences between humans and machines - we contribute to the growing field of HMC from a critical perspective. The consequences of an "othering" discourse and ableist communication scripts, as illustrated in $D B H$, should encourage scholars to examine what unjust discourses, oppressive communication scripts, and unseen forms of control might exist in our own society. Additionally, as Craig et al. (2020) note in their exploratory study of character attachment in $D B H$, "perceptions held about video game robots could have importance for social robots encountered in daily living" (p. 170). As such, we draw the following conclusions from our application of ableism as an analytic sensibility to the ways in which humans make sense of machineothers.

Beyond gender stereotypes, there is a need for researchers to further explore how humans apply human-human scripts to human-machine interactions, especially if these scripts may render the machine as less-than or anxiety-arousing. At this point, it is unclear whether the mindless application of oppressive social scripts is limited to initial interactions with machines, or how the various factors described by Gambino et al. (2020) impact pervasive social scripts like ableism. Gambino et al. make a case for an extended CASA, arguing that people have changed given that their experience with and knowledge of media agents and machines have increased dramatically. Future studies should explore how experience with machines impacts the mindless application of oppressive human-to-human scripts. As technology advances and becomes more human-like (Gambino et al., 2020), it would be interesting to examine how higher levels of anthropomorphism impact the tendency to apply ableist frameworks. As we described in our analysis of $D B H$, high levels of anthropomorphism could invite high levels of ableism and cyborg anxiety that manifest in a desire for control. Designers of machines may find it challenging to avoid the application of human-to-human scripts while advancing technology.

We live in a technological era where "ongoing and long-term interactions [with machines] present the opportunity for individuals to develop relationships with media agents similar to those with humans" (Gambino et al., 2020, p. 77). One way to overcome the mindless application of ableist scripts to HMC could be increased exposure to and experience with machines as communicative subjects, resulting in shifting the interaction between humans and machines from an I-It perspective to an I-Thou perspective (Westerman et al., 2020). As humans engage more frequently and in more depth with machines, they may realize that certain human-human scripts may not be as applicable to humanmachine contexts as they thought, prompting them to develop scripts for human-media agent interaction (Gambino et al., 2020).

Further, we also recognize that studying how humans treat human-machine interactions as I-It may also be valuable for understanding HHC. Indeed, as Westerman et al. (2020) conclude, "it seems like we respond to machines and AI as we do people, but we may not always respond to people in a very interpersonal way" (p. 403). The scripts humans use to make sense of human-machine interactions are undeniably layered, and a deeper dive may reveal how and why "we may treat people like computers" (Gambino et al., 2020, p. 79). Therefore, insights gained in an HMC context about how social scripts such as ableism are applied mindlessly may contribute to our understanding of how ableism and other pervasive social scripts play out in human-human interactions. Posthuman and critical disability 
studies of ableism contribute not only to our understanding of machines, but also to our understanding of what it means to be human. As Guzman (2018) reminds us, "Communication, even with machines, shapes the Self" (p. 20). Thus, engaging with machines as communicative subjects may not only lead to rewriting the communicative scripts we rely on to make sense of our communicative others and our interaction with them, but also sheds light on what it means to be(come) human in a world increasingly full of (humanoid) machines.

\section{Author Biographies}

Marco Dehnert (MA, Arizona State University) is a doctoral student in the Hugh Downs School of Human Communication at Arizona State University. His research interests focus on critical/cultural approaches to communication and rhetorical studies. With a broad focus on how one recognizes and disavows the Other, his work is situated at the intersections of critical and intersectional rhetorics, queer and transgender communication studies, qualitative inquiry, and human-machine communication.

\section{https://orcid.org/0000-0002-7456-0743}

Rebecca B. Leach (MS, Texas Christian University) is a doctoral student in the Hugh Downs School of Human Communication at Arizona State University. Her research examines the factors that foster flourishing in organization and society, including compassion, resilience, justice, and inclusion.

\section{https://orcid.org/0000-0002-0606-9549}

\section{References}

Cherney, J. L. (2019). Ableist rhetoric: How we know, value, and see disability. The Pennsylvania State University Press.

Christoph, K., Dorothée, H., \& Peter, V. (2009). The video game experience as "true" identification: A theory of enjoyable alterations of players' self-perception. Communication Theory, 19(4), 351-373. https://doi.org/10.1111/j.1468-2885.2009.01347.x

Cialdini, R. B. (2009). Influence: Science and practice (5th ed.). Pearson Education.

Craig, M. J., Edwards, C., \& Edwards, A. (2020). "But they're my avatar": Examining character attachment to android avatars in Quantic Dream's Detroit: Become Human. HRI '20: Companion of the 2020 ACM/IEEE International Conference on Human-Robot Interaction, 169-170. https://doi.org/10.1145/3371382.3378298

Edwards, A., Edwards, C., Westerman, D., \& Spence, P. R. (2019). Initial expectations, interactions, and beyond with social robots. Computers in Human Behavior, 90, 308-314. https://doi.org/10.1016/j.chb.2018.08.042

Edwards, A. P. (2018). Animals, humans, and machines: Interactive implications of ontological classification. In A. L. Guzman (Ed.), Human-machine communication: Rethinking communication, technology, and ourselves (pp. 29-49). Peter Lang.

Edwards, R. C. (1978). The social relations of production at the point of production. Insurgent Sociologist, 8(2-3), 109-125. https://doi.org/10.1177/089692057800800213 
Fortunati, L., Cavallo, F., \& Sarrica, M. (2020). Multiple communication roles in humanrobot interactions in public space. International Journal of Social Robotics, 12(4), 931944. https://doi.org/10.1007/s12369-018-0509-0

Fortunati, L., \& Edwards, A. (2020). Opening space for theoretical, methodological, and empirical issues in human-machine communication. Human-Machine Communication, 1, 7-18. https://doi.org/10.30658/hmc.1.1

Gambino, A., Fox, J., \& Ratan, R. A. (2020). Building a stronger CASA: Extending the computers are social actors paradigm. Human-Machine Communication, 1, 71-85. https:// doi.org/10.30658/hmc.1.5

Giddens, A. (1984). The constitution of society: Outline of the theory of structuration. University of California Press.

Goodley, D. (2014). Dis/ability studies: Theorising disablism and ableism. Routledge.

Goodley, D., Lawthom, R., Liddiard, K., \& Runswick-Cole, K. (2019). Provocations for critical disability studies. Disability \& Society, 34(6), 972-997. https://doi.org/10.1080/ 09687599.2019.1566889

Goodley, D., Lawthom, R., \& Runswick-Cole, K. (2014). Posthuman disability studies. Subjectivity, 7(4), 342-361. https://doi.org/10.1057/sub.2014.15

Gossett, L. M. (2009). Organizational control theory. In S. W. Littlejohn \& K. A. Foss (Eds.), Encyclopedia of communication theory (pp. 706-710). SAGE.

Gunkel, D. J. (2012). Communication and artificial intelligence: Opportunities and challenges for the 21st century. Communication +1, 1(1), 1-25. https://doi.org/10.7275/ R5QJ7F7R

Guzman, A. L. (2018). Introduction: "What is human-machine communication, anyway?" In A. L. Guzman (Ed.), Human-machine communication: Rethinking communication, technology, and ourselves (pp. 1-28). Peter Lang.

Guzman, A. L. (2020). Ontological boundaries between humans and computers and the implications for human-machine communication. Human-Machine Communication, 1, 37-54. https://doi.org/10.30658/hmc.1.3

Hamraie, A., \& Fritsch, K. (2019). Crip technoscience manifesto. Catalyst: Feminism, Theory, Technoscience, 5(1), 1-34. https://doi.org/10.28968/cftt.v5i1.29607

Johnson, J., \& Kennedy, K. (2020). Introduction: Disability, in/visibility, and risk. Rhetoric Society Quarterly, 50(3), 161-165. https://doi.org/10.1080/02773945.2020.1752126

Krämer, N. C., von der Pütten, A., \& Eimler, S. (2012). Human-agent and human-robot interaction theory: Similarities to and differences from human-human interaction. In M. Zacarias \& J. V. Oliveira (Eds.), Human-computer interaction: The agency perspective (pp. 215-240). Springer.

Leach, R., \& Dehnert, M. (in press). Becoming the other: Examining race, gender, and sexuality in Detroit: Become Human. Review of Communication. https://doi.org/10.1080/15 358593.2021.1892173

Makkawy, A., \& Moreman, S. T. (2019). Putting crip in the script: A critical communication pedagogical study of communication theory textbooks. Communication Education, 68(4), 401-416. https://doi.org/10.1080/03634523.2019.1643898

McRuer, R. (2006). Crip theory: Cultural signs of queerness and disability. New York University Press. 
Meekosha, H., \& Shuttleworth, R. (2009). What's so 'critical' about critical disability studies? Australian Journal of Human Rights, 15(1), 47-75. https://doi.org/10.1080/13232 38X.2009.11910861

Mik-Meyer, N. (2016). Othering, ableism and disability: A discursive analysis of coworkers' construction of colleagues with visible impairments. Human Relations, 69(6), 1341-1363. https://doi.org/10.1177/0018726715618454

Moreman, S. T., \& McIntosh, D. M. (2010). Brown scriptings and rescriptings: A critical performance ethnography of Latina drag queens. Communication and Critical/Cultural Studies, 7(2), 115-135. https://doi.org/10.1080/14791421003767912

Mori, M. (2012). The uncanny valley (K. F. MacDorman \& N. Kageki, Trans.). IEEE Robotics \& Automation Magazine, 19(2), 98-100. https://doi.org/10.1109/MRA.2012.2192811

Nass, C., \& Moon, Y. (2000). Machines and mindlessness: Social responses to computers. Journal of Social Issues, 56(1), 81-103. https://doi.org/10.1111/0022-4537.00153

Peter, J., \& Kühne, R. (2018). The new frontier in communication research: Why we should study social robots. Media and Communication, 6(3), 73-76. https://doi.org/10.17645/ mac.v6i3.1596

Quantic Dream. (2018). Detroit: Become human [Video game]. Paris, France: Sony Interactive Entertainment.

Reeve, D. (2012). Cyborgs, cripples and iCrip: Reflections on the contribution of Haraway to disability studies. In D. Goodley, B. Hughes, and L. J. Davis (Eds.), Disability and social theory: New developments and directions (pp. 91-111). Palgrave Macmillan.

Reynolds, J. M. (2017). "I'd rather be dead than disabled"-The ableist conflation and the meanings of disability. Review of Communication, 17(3), 149-163. https://doi.org/10.10 80/15358593.2017.1331255

Sandry, E. (2018). Aliveness and the off-switch in human-robot relations. In A. L. Guzman (Ed.), Human-machine communication: Rethinking communication, technology, and ourselves (pp. 51-66). Peter Lang.

Shew, A. (2020). Ableism, technoableism, and future AI. IEEE Technology and Society Magazine, 39(1), 40-85. https://doi.org/10.1109/MTS.2020.2967492

Swartz, L., \& Watermeyer, B. (2008). Cyborg anxiety: Oscar Pistorius and the boundaries of what it means to be human. Disability \& Society, 23(2), 187-190. https://doi. org/10.1080/09687590701841232

Tompkins, P. K., \& Cheney, G. (1985). Communication and unobtrusive control in contemporary organizations. In R. D. McPhee \& P. K. Tompkins (Eds.), Organizational communication: Traditional themes and new directions (pp. 179-210). SAGE.

Van Trigt, P., Kool, J., \& Schippers, A. (2016). Humanity as a contested concept: Relations between disability and 'being human' (Editorial). Social Inclusion, 4(4), 125-128. https:// doi.org/10.17645/si.v4i4.754

Vehmas, S., \& Watson, N. (2014). Moral wrongs, disadvantages, and disability: A critique of critical disability studies. Disability \& Society, 29(4), 638-650. https://doi.org/10.108 0/09687599.2013.831751

Westerman, D., Edwards, A. P., Edwards, C., Luo, Z., \& Spence, P. R. (2020). I-It, I-Thou, I-Robot: The perceived humanness of AI in human-machine communication. Communication Studies, 71(3), 393-408. https://doi.org/10.1080/10510974.2020.1749683 
Wolbring, G. (2009). What next for the human species? Human performance enhancement, ableism and pluralism. In N. Hällström (Ed.), Development Dialogue, 52 (pp. 141161). Dag Hammarskjöld Foundation. http://www.daghammarskjold.se/wp-content/ uploads/2009/DD52_web.pdf

Wright, B. M., \& Barker, J. R. (2000). Assessing concertive control in the term environment. Journal of Occupational \& Organizational Psychology, 73(3), 345-361. https://doi. org/10.1348/096317900167065 